(C) The Author(s), 2021. Published by Cambridge University Press on behalf of The Nutrition Society. This is an Open Access article, distributed under the terms of the Creative Commons Attribution licence (http://creativecommons.org/licenses/by/4.0/), which permits unrestricted re-use, distribution, and reproduction in any medium, provided the original work is properly cited.

\title{
Malnutrition in early life and its neurodevelopmental and cognitive consequences: a scoping review
}

\author{
A. Suryawan ${ }^{1}$ (1) , M.Y. Jalaludin ${ }^{2}$, B.K. Poh ${ }^{3}$, R. Sanusi ${ }^{4}$, V.M.H. Tan ${ }^{5}$, J.M. Geurts ${ }^{6}$ and L. Muhardi ${ }^{7}$ (1) \\ ${ }^{1}$ Department of Child Health, Faculty of Medicine, Universitas Airlangga, Soetomo General Academic Hospital, Surabaya, \\ Indonesia \\ ${ }^{2}$ Department of Paediatrics, Faculty of Medicine, University Malaya, 59100 Kuala Lumpur, Malaysia \\ ${ }^{3}$ Nutritional Sciences Programme and Centre for Community Health Studies (ReaCH), Faculty of Health Sciences, Universiti \\ Kebangsaan Malaysia, Kuala Lumpur, Malaysia \\ ${ }^{4}$ Department of Human Nutrition and Dietetics, University of Ibadan, Nigeria \\ ${ }^{5}$ Dietetics and Nutrition Programme, Health and Social Sciences cluster, Singapore Institute of Technology, Singapore \\ ${ }^{6}$ FrieslandCampina, Amersfoort, The Netherlands \\ ${ }^{7}$ FrieslandCampina AMEA, Singapore
}

\section{Abstract}

The negative impact of stunting and severe underweight on cognitive neurodevelopment of children is well documented; however, the effect of overweight/obesity is still unclear. The 2018 Global Nutrition Report reported that stunting and overweight concurrently affect 189 million children worldwide. As existing reviews discuss undernutrition and overweight/obesity separately, this scoping review aims to document the impact of mild/moderate and severe underweight, stunting, and overweight/obesity among children aged 0-60 months on their cognitive neurodevelopmental trajectories. Twenty-six articles were analysed to extract significant information from literature retrieved from PubMed and Cochrane databases published from 1 January 2009 to 31 October 2019. Length gain is associated with cognitive neurodevelopment in normo-nourished and stunted children aged under 24 months. Among stunted children, it seems that cognitive and neurodevelopmental deficits can potentially be recovered before 8 years of age, particularly in those whose nutritional status has improved. The impact of overweight/obesity on cognitive neurodevelopment appears to be limited to attention, gross motor skills and executive control. Parental education level, birth weight/length, breastfeeding duration, and sanitation level are some identifiable factors that modify the impact of undernutrition and overweight/obesity on cognitive and neurodevelopment. In conclusion, underweight, stunting and overweight/obesity have a significant impact on cognitive neurodevelopment. Multidimensional approaches with various stakeholders should address all issues simultaneously, such as improving sanitation levels, assuring parental job security and adequate social welfare, and providing access to adequate nutrients for catch-up growth among underweight or stunted children and to affordable healthy foods for those who are overweight/obese and from low socio-economic status.

Key words: Underweight: Stunting: Obesity: Cognition: Neurodevelopment

(Received 5 May 2020; revised 26 May 2021; accepted 31 May 2021; accepted manuscript published online 8 June 2021)

\section{Introduction}

Malnutrition, including wasting or low weight-for-height, stunting or low height-for-age, and underweight or low weight-forage, micronutrient deficiencies, and overweight/obesity, are public health problems that can affect children aged less than 60 months. While the number of stunted children worldwide has decreased in recent years, the number of overweight children has increased, and the total number of children affected by stunting and overweight is approximately 189 million $^{(1)}$. The World Health Organization (WHO) in 2019 reported that undernutrition and overweight/obesity coexist together in more than one-third of low- and middle-income countries ${ }^{(2)}$. Given the potential for both ends of the malnutrition spectrum to affect neurodevelopment, it is important to consider them together.

Malnutrition leads to economic, social and health issues for families, at the community and national level ${ }^{(3)}$. More importantly, severe undernutrition - defined as severe stunting (below height-for-age $z$-score (HAZ) minus three standard deviations $(-3 \mathrm{SD})$ ) and severe underweight (below weight-for-age $z$-score (WAZ) minus three standard deviations $(-3 \mathrm{SD}))^{(4)}$ - impair children's development in the short (delayed cognitive, behavioural and motor development), medium (lower intelligence

Corresponding author: Muhammad Yazid Jalaludin, Professor, Department of Paediatrics, Faculty of Medicine, University of Malaya, 59100 Kuala Lumpur, Malaysia. Tel.: 03-79492065, Fax: 03-79494704, Email: yazidj@ummc.edu.my or yazidjal@um.edu.my 
quotient (IQ) and school/academic achievement) and long term (human capital), mostly in low- and middle-income countries ${ }^{(5-8)}$. It is currently unclear whether overweight and obesity impact neurodevelopment and cognition or, vice versa, whether neurodevelopmental deficits impact overweight and obesity, or both.

Despite the reduction in stunting over the last decade ${ }^{(1)}$, there still exists a need to better understand if there are opportunities to mitigate the negative, long-term impact of stunting on human capital development. It is also currently unclear whether interventions can be targeted at specific aspects of neurodevelopment and cognition, taking into consideration the timing of malnutrition with the timing of cognitive neurodevelopment in early childhood. More information is also needed to understand the aspects of neurodevelopment and cognition that are affected among overweight/obese children in order to design specific interventions.

As most existing reviews discuss undernutrition and overweight/obesity separately, this scoping review was designed to extract information on both conditions from the published literature, to study their impact as compared with normonourished children aged 0-60 months on cognitive neurodevelopmental aspects (cognition, behavioural, motor and emotional development) in early, mid and later life. Undernutrition is defined as stunting (HAZ $<-2 \mathrm{SD}$ ) and underweight (WAZ $<-2 \mathrm{SD}$ ), while overweight is defined as $2 \mathrm{SD}>\mathrm{WAZ}<3 \mathrm{SD}$ and obesity as $W A Z \geq 3 \mathrm{SD})^{(4)}$. This combined approach will conceivably allow for a more holistic view of the situation commonly found in many countries across the world.

\section{Methods}

This review followed the consistent approach of a scoping review to compile findings from studies with different designs and methodologies to answer the stated critical research questions $^{(9)}$. The research questions are as follows: (i) Which developmental aspects are affected by obesity/overweight and undernutrition during the first 60 months of life? (ii) What other factors can modify these outcomes? (iii) Which factors can mitigate the impact of obesity/overweight and undernutrition on neurodevelopment and cognition? (iv) Is the effect on cognitive neurodevelopment partly or fully reversible? and (v) What are the implications for intervention strategies?

Literature published from 1 January 2009 to 31 October 2019 in the PubMed and Cochrane databases was retrieved using formulated search terms. The title and abstract of each article retrieved were screened based on the inclusion criteria by all authors. A simple grading of controlling study biases using JADAD criteria for randomised controlled studies, Newcastle Ottawa Scale for cohort studies, and National Heart, Lung and Blood Institute (NHLBI) criteria for observational cross-sectional studies were then conducted by five authors (A.S., M.Y.J., P.B.K., L.M. and J.G.) to select studies for further qualitative analyses (Tables 1 and 2). References of relevant narrative reviews (cross-references) were also examined to search for further studies that met the inclusion criteria. J.G. and L.M. then independently extracted data from the identified full-text articles.
Data extraction tables incorporated important information from eligible full texts to allow the extraction of significant information for the review.

\section{Results and discussion}

\section{Descriptive summary of included studies}

From the ninety-one articles originally identified through the search strategy, twenty-six full-text articles were selected after a review of cross-references in eligible narrative reviews. The full text was used for information extraction and grading for quality assessment (Fig. 1).

Information was categorised based on the type of study and nutritional status of the children (Tables 1 and 2) as well as by factors influencing the impact of nutritional status on cognitive neurodevelopment (Table 3). Information was extracted from fourteen observational studies (undernutrition ( $n=8$ articles), normo-nutrition ( $n=5$ articles) and overweight/obesity ( $n=1$ article), four intervention trials (undernutrition $(n=2$ articles) and normo-nutrition ( $n=2$ articles)), four systematic reviews (undernutrition ( $n=1$ article), normo-nutrition $(n=2$ articles) and overweight/obesity ( $n=2$ articles)) and four meta-analyses (all on undernutrition). All publications on underand normo-nourished children were conducted in low- to middle-income countries, while all articles about overweight/ obese children reported data from high-income countries. No intervention study was retrieved on overweight/obese children. Approximately $85 \%(n=22)$ of the retrieved literature had good quality in controlling bias.

To the best of our knowledge, this review complements previous meta-analyses and systematic reviews ${ }^{(6-8)}$ and includes a wider spectrum of suboptimal nutritional status (ranging from mild/moderate to severe underweight and stunting to overweight/obesity) during the first 60 months of life on various neurodevelopment aspects (cognition, motor development, temperament, socio-economic status, and academic achievement) that manifest from infancy through school-age to adulthood (Fig. 2). This review also covers observational longitudinal cohorts and interventional studies with nutrition- and non-nutrition-related interventions on the outcomes of interest.

\section{The importance of monitoring weight, length and head circumference during the first 24 months of age}

From the reviewed studies, length-for-age $z$-scores (LAZ) and height-for-age $z$-scores (HAZ) (anthropometric measures of stunting in children) were associated with various elements of cognitive functioning, such as attention span, time to proper walking, mathematics and language abilities in early life, and income and choice of partner/age at marriage in later life (Table 1). It was reported that healthy infants in the highest quartile of neonatal weight-, length- and head circumference-gain during the first 4 weeks of life had higher IQ scores at 6 years of age than those in the lowest quartile ${ }^{(10)}$.

From a longitudinal study in the Philippines, regardless of HAZ categories at 6 months (defined as moderate/severe stunting ( $\mathrm{HAZ}<-2$ ), mild stunting $(-2 \leq \mathrm{HAZ}<-1)$, at risk of stunting $(-1<\mathrm{HAZ}<0)$, or normal height $(\mathrm{HAZ} \geq 0)$ ), more 
Table 1. Key findings from retrieved observational studies on growth and the impact on neurodevelopment and cognition

\begin{tabular}{|c|c|c|c|c|c|c|c|c|}
\hline & First author, year of publication & $\begin{array}{l}\text { Country of } \\
\text { study }\end{array}$ & $\begin{array}{l}\text { Sample size and age at } \\
\text { enrolment }\end{array}$ & $\begin{array}{l}\text { Type of study (longitudinal } \\
\text { cohort, cross-sectional, sys- } \\
\text { tematic review, or meta- } \\
\text { analysis) }\end{array}$ & $\begin{array}{l}\text { Nutritional status of } \\
\text { the study population }\end{array}$ & $\begin{array}{c}\text { Developmental tools and age at } \\
\text { assessment }\end{array}$ & $\begin{array}{l}\text { Affected cognitive and } \\
\text { neurodevelopmental } \\
\text { aspects by suboptimal } \\
\text { growth }\end{array}$ & $\begin{array}{l}\text { Study } \\
\text { quality }\end{array}$ \\
\hline 1. & Sudfeld CR, $2015^{(6)}$ & $\begin{array}{l}\text { Low- and } \\
\text { middle- } \\
\text { income } \\
\text { countries }\end{array}$ & $\begin{array}{l}\text { Children aged }<12 \text { years } \\
\text { (children aged }>5-12 \\
\text { years were excluded } \\
\text { from the present } \\
\text { review) }\end{array}$ & $\begin{array}{l}\text { Systematic review from } \\
\text { longitudinal cohorts and } \\
\text { surveys }\end{array}$ & General population & $\begin{array}{l}\text { During the first } 60 \text { months of life: } \\
\text { - Cognitive } \\
\text { - Motor } \\
\text { - Socio-emotional }\end{array}$ & $\begin{array}{l}\text { - General cognition } \\
\text { - Odds of walking } \\
\text { - Motor score } \\
\text { - Social skills }\end{array}$ & Good \\
\hline 2. & Hamadani JD, 2014 $4^{(12)}$ & Bangladesh & 2853 singletons & $\begin{array}{l}\text { Longitudinal cohort sub sam- } \\
\text { ple from birth to } 64 \\
\text { months as part of a larger } \\
\text { maternal interventional } \\
\text { study }\end{array}$ & General population & $\begin{array}{l}\text { - Children's problem-solving ability mea- } \\
\text { sured using two } 1 \text {-step means-end tests } \\
\text { 'Support' and 'Cover' at } \\
7 \text { months } \\
\text { - Mental development index (MDI) mea- } \\
\text { sured using the Bayley Scales of Infant } \\
\text { Development Revised version at } 18 \\
\text { months } \\
\text { - Children's IQ measured using the } \\
\text { Wechsler Preschool and Primary Scale } \\
\text { of Intelligence (WPPSI) at } 64 \text { months }\end{array}$ & IQ score & Good \\
\hline 3. & Camargo-Figuera FA, 2014(10) & Brazil & 4231 singletons & $\begin{array}{l}\text { Longitudinal cohort from } \\
\text { birth to } 6 \text { years of age }\end{array}$ & General population & - WISC-III at 72 months & IQ score & Good \\
\hline 4. & Smithers LG, $2013^{(39)}$ & Belarus & 16692 children & $\begin{array}{l}\text { Longitudinal cohort from } \\
\text { birth to } 1 \text { year from a sub- } \\
\text { sample from PROBIT Trial }\end{array}$ & General population & $\begin{array}{l}\text { At } 6.5 \text { years: } \\
\text { - Cognitive ability using Wechsler } \\
\text { Abbreviated Scales of Intelligence } \\
\text { - Child behaviour using the Strengths and } \\
\text { Difficulties Questionnaire }\end{array}$ & IQ score & Good \\
\hline 5. & Aubuchon-Endsley NL, 2011(40) & Ethiopia & 108 infants & $\begin{array}{l}\text { Longitudinal cohort from } 6 \\
\text { months to } 9 \text { months of } \\
\text { age }\end{array}$ & Healthy population & $\begin{array}{l}\text { - Temperament and attention assessed } \\
\text { using modified version of the Laboratory } \\
\text { Temperament Assessment Battery } \\
\text { (Lab-TAB) and MOFPTask at } 6 \text { and } 9 \\
\text { months }\end{array}$ & $\begin{array}{l}\text { - Duration and num- } \\
\text { ber of inattention } \\
\text { periods } \\
\text { - Mean of looking time } \\
\text { - Total duration of } \\
\text { looking }\end{array}$ & Poor \\
\hline 6. & Mohd Nasir MT, 2012 ${ }^{(14)}$ & Malaysia & $\begin{array}{l}1933 \text { pre-schoolers aged } \\
4-6 \text { years }\end{array}$ & Cross-sectional study & Healthy population & $\begin{array}{l}\text { - Raven's Coloured Progressive Matrices } \\
\text { at } 48-72 \text { months }\end{array}$ & $\begin{array}{l}\text { Cognitive perfor- } \\
\text { mance }\end{array}$ & Good \\
\hline 7 & Miller AC, $2015^{(8)}$ & $\begin{array}{l}\text { Low-middle } \\
\text { income } \\
\text { countries }\end{array}$ & $\begin{array}{l}58513 \text { children aged } \\
36-59 \text { months }\end{array}$ & $\begin{array}{l}\text { Meta-analysis from } 15 \\
\text { Multiple Indicator Cluster } \\
\text { Surveys results (MICS-4) }\end{array}$ & Undernutrition & $\begin{array}{l}\text { Early Childhood Development Index } \\
\text { scores for children aged } 36-59 \text { months } \\
\text { old }\end{array}$ & $\begin{array}{l}\text { - Overall development } \\
\text { and learning } \\
\text { capabilities } \\
\text { - Literacy skills } \\
\text { - Numerical skills }\end{array}$ & Good \\
\hline 8 & Nguyen $\mathrm{PH}, 2018^{(29)}$ & Vietnam & 1458 children & $\begin{array}{l}\text { Cohort study from offspring } \\
\text { of the first phase study (a } \\
\text { randomised controlled trial } \\
\text { of pre-conceptional micro- } \\
\text { nutrient supplementation } \\
\text { (PRECONCEPT study) } \\
\text { from birth until } 2 \text { years of } \\
\text { age }\end{array}$ & General population & $\begin{array}{l}\text { Bayley Scales of Infant } \\
\text { Development-III } \\
\text { at } 12 \text { and } 24 \text { months }\end{array}$ & $\begin{array}{l}\text { - Cognitive } \\
\text { development } \\
\text { - Language } \\
\text { development } \\
\text { - Motor development }\end{array}$ & Good \\
\hline
\end{tabular}


Table 1. (Continued)

\begin{tabular}{|c|c|c|c|c|c|c|c|c|}
\hline & First author, year of publication & $\begin{array}{l}\text { Country of } \\
\text { study }\end{array}$ & $\begin{array}{c}\text { Sample size and age at } \\
\text { enrolment }\end{array}$ & $\begin{array}{l}\text { Type of study (longitudinal } \\
\text { cohort, cross-sectional, sys- } \\
\text { tematic review, or meta- } \\
\text { analysis) }\end{array}$ & $\begin{array}{l}\text { Nutritional status of } \\
\text { the study population }\end{array}$ & $\begin{array}{c}\text { Developmental tools and age at } \\
\text { assessment }\end{array}$ & $\begin{array}{l}\text { Affected cognitive and } \\
\text { neurodevelopmental } \\
\text { aspects by suboptimal } \\
\text { growth }\end{array}$ & $\begin{array}{c}\text { Study } \\
\text { quality }\end{array}$ \\
\hline 9 & Casale D, 2014 ${ }^{(19)}$ & South Africa & 3273 children & $\begin{array}{l}\text { Cohort study from the Birth } \\
\text { to Twenty cohort study } \\
\text { from } 2 \text { years to } 5 \text { years } \\
\text { of age }\end{array}$ & Stunted & $\begin{array}{l}\text { - At } 48 \text { months: social skills competence or } \\
\text { 'daily living skills' assessed using VSMS } \\
\text { - At } 60 \text { months: cognitive functioning } \\
\text { assessed using R-DPDQ }\end{array}$ & $\begin{array}{l}\text { - Cognitive functioning } \\
\text { - Social skills } \\
\text { competence } \\
\text { - Fine motor skills }\end{array}$ & Good \\
\hline 10 & Crookston BT, 2013 $3^{(16)}$ & $\begin{array}{l}\text { - Ethiopia } \\
\text { - India } \\
\text { - Peru } \\
\text { - Vietnam }\end{array}$ & 8062 infants & $\begin{array}{l}\text { Cohort of multi-country stud- } \\
\text { ies from } 1 \text { year to } 8 \text { years } \\
\text { of age }\end{array}$ & $\begin{array}{l}\text { Stunted and healthy } \\
\text { children }\end{array}$ & $\begin{array}{l}\text { At } 8 \text { years: } \\
\text { - Schooling average } \\
\text { - Mathematics test } \\
\text { - Reading comprehension/verbal achieve- } \\
\text { ment: Early Grade Reading Assessment } \\
\text { - Receptive vocabulary: Peabody Picture } \\
\text { Vocabulary Test (PPVT) }\end{array}$ & $\begin{array}{l}\text { - Overall schooling } \\
\text { - Receptive vocabu- } \\
\quad \text { lary } \\
\text { - Mathematics score }\end{array}$ & Good \\
\hline 11 & Cheung YB, 2010(11) & Philippines & 1516 infants & $\begin{array}{l}\text { Cohort study from the 'Cebu } \\
\text { Longitudinal } \\
\text { Health and Nutrition Survey' } \\
\text { from } 6 \text { to } 24 \text { months old } \\
\text { followed up at age } \\
11 \text { years }\end{array}$ & $\begin{array}{l}\text { Stunted and Healthy } \\
\text { children }\end{array}$ & $\begin{array}{l}\text { At } 11 \text { years } \\
\text { - Non-verbal intelligence test } \\
\text { - English reading comprehension test } \\
\text { - Mathematics test }\end{array}$ & Cognitive ability & Good \\
\hline 12 & Hoddinott J, 2013 $3^{(17)}$ & Guatemala & 1338 adults & $\begin{array}{l}\text { Retrospective cohort aged } \\
25-42 \text { years who were } \\
\text { studied as 24-month } \\
\text { infants in 1969-1977 }\end{array}$ & Healthy adults & $\begin{array}{l}\text { - Age of starting and leaving school } \\
\text { - Highest grade attained } \\
\text { - Inter-American Reading Series (Serie } \\
\text { Interamericana) vocabulary (Level 3) } \\
\text { - Reading comprehension (Level 2) test } \\
\text { modules } \\
\text { - Nonverbal cognitive ability: assessed } \\
\text { using Raven's Standard Progressive } \\
\text { Matrices test }\end{array}$ & $\begin{array}{l}\text { - Overall schooling } \\
\text { - Reading scores } \\
\text { - Non-verbal cognitive } \\
\text { scores } \\
\text { - Characteristics of } \\
\text { marriage partners; } \\
\text { older age at first } \\
\text { birth; a smaller } \\
\text { number of pregnan- } \\
\text { cies and births } \\
\text { - Household per cap- } \\
\text { ita income } \\
\text { - Adult health }\end{array}$ & Good \\
\hline 13 & Waber DP, 2014 ${ }^{(41)}$ & Barbados & 77 adults & $\begin{array}{l}\text { Retrospective cohort study } \\
\text { from an Intervention pro- } \\
\text { gram with history of infan- } \\
\text { tile malnutrition in the first } \\
\text { year of life and healthy } \\
\text { controls born between } \\
1967 \text { and } 1972\end{array}$ & $\begin{array}{l}\text { Grade II-III protein } \\
\text { energy malnutrition } \\
\text { and healthy popu- } \\
\text { lation }\end{array}$ & $\begin{array}{l}\text { - Adult IQ: Wechsler Abbreviated Scale of } \\
\text { Intelligence - Vocabulary and Matrix } \\
\text { Reasoning subtests } \\
\text { - Academic Achievement: Wide Range } \\
\text { Achievement Test-III - Reading, } \\
\text { Spelling, and Calculation subtests }\end{array}$ & IQ score & Good \\
\hline 14 & Baker-Henningham H, 2009(15) & Bangladesh & $\begin{array}{l}212 \text { undernourished chil- } \\
\text { dren and } 108 \text { normo- } \\
\text { nourished controls }\end{array}$ & $\begin{array}{l}\text { Cross-sectional study of chil- } \\
\text { dren aged 6-24 months }\end{array}$ & $\begin{array}{l}\text { Underweight (weight- } \\
\text { for-age } z \text {-score } \\
<-2 \text { ) and } \\
\text { normo-nourished } \\
\text { children }\end{array}$ & $\begin{array}{l}\text { At 6-24 months: } \\
\text { - Baseline assessment using revised } \\
\text { version of the Bayley Scales of Infant } \\
\text { Development } \\
\text { - Temperament assessed through an inter- } \\
\text { viewer-administered maternal question- } \\
\text { naire consisting of seven subscales }\end{array}$ & $\begin{array}{l}\text { - Social skills } \\
\text { - Attention } \\
\text { - Fear } \\
\text { - Negative emotion }\end{array}$ & Good \\
\hline
\end{tabular}


Table 1. (Continued)

Type of study (longitudinal cohort, cross-sectional, sys-

First author, year of publication

Country of study

15 Sudfeld CR, 2015(42) Tanzania

Sample size and age at enrolment analysis)

1036 infants aged 18-36 months

Cross-sectional study on existing data from a cohort

$\begin{array}{cc}\text { Systematic } & \text { Pre-school children }(+ \\ \text { review of } & \text { adolescents aged 18 } \\ 67 \text { studies } & \text { years and under, } \\ \text { in high- } & \text { excluded from the } \\ \text { income } & \text { present review) }\end{array}$

countries

1- to 5-year children (+ adolescents and adults

aged up to 21 years,

excluded from the

present review)

18 Morano M, 2011(24)

Italy

Overweight children ( $n$

$=38$ ) and non-over-

weight children $(n=42)$

with a mean age of $4+$

Cross-sectional study

ystematic review from observational cross-sectional and longitudinal studies

0.5 year
Systematic review of four observational studies
Affected cognitive and

neurodevelopmental

aspects by suboptimal Study

Nutritional status of

Developmental tools and age at growth quality

General population

Bayley Scales of Infant Development III at 18-36 months:

- Cognition

- Communication (comprising expressive

and receptive communication skills)

- Motor (comprising fine and gross motor

$$
\text { skills) }
$$

- Self-regulation (laboratory tasks)

- Motor skills (Zurich Neuromotor

Assessment Test; Motor Test Battery)

- Verbal ability (Peabody Picture

Vocabulary Test)

- Concentration (Frankfurter Test für

Fünfjährige - Konzentration)

- Intelligence (Culture Fair Test)

- Locomotor skills, object-control skills (Test of Gross Motor Development)

- Delay of gratification (Mischel and

Ebbesen's delay of gratification waiting task)

- Movement skills

- Cognitive develop- Good

ment

Communication

skills

- Motor development

- General intellectual Poor

- Executive control

- Delayed gratification

- Inhibition strategy

- Learning and

memory

gallop, hop, leap, horizontal jump, skip and slide) and five object-control skills (two-hand strike, stationary bounce,

catch, kick and overhand throw) at age of enrolment
- Delay of Gratification Task, Self-Control

Task

- Children's Behaviour Questionnaire

- Classroom Engagement

- Social Behaviour Questionnaire

- Go-No Go Task

- Behavioural Rating Inventory of

Executive Functioning (self-report)

- Incompatibility Task of Attention

Assessment Battery

- Test of Gross Motor Development

Quotient to assess seven locomotor

skills (run,

Inhibitory control

Emotional regulation

healthy

controls

Motor competence 
Table 2. Key findings from retrieved interventional studies on undernutrition and the impact on neurodevelopment and cognition

\begin{tabular}{|c|c|c|c|c|c|c|c|c|c|c|}
\hline No. & $\begin{array}{l}\text { First author, year } \\
\text { of publication }\end{array}$ & Country of study & $\begin{array}{l}\text { Sample size and } \\
\text { age at enrolment }\end{array}$ & $\begin{array}{l}\text { Type of } \\
\text { studies }\end{array}$ & Type of intervention & $\begin{array}{l}\text { Nutritional } \\
\text { status of the } \\
\text { study popula- } \\
\text { tion }\end{array}$ & $\begin{array}{l}\text { Developmental tools } \\
\text { and age at assessment }\end{array}$ & Key findings & $\begin{array}{l}\text { Affected cognitive } \\
\text { and neurodevelop- } \\
\text { mental aspects by } \\
\text { suboptimal growth }\end{array}$ & $\begin{array}{l}\text { Study } \\
\text { quality }\end{array}$ \\
\hline 1 & Prado EL, $2019^{(7)}$ & $\begin{array}{l}\text { Low- to high- } \\
\text { income coun- } \\
\text { tries }\end{array}$ & $\begin{array}{l}72275 \text { children } \\
\text { aged } 0-60 \\
\text { months }\end{array}$ & $\begin{array}{l}\text { Meta-analysis } \\
\text { from } 52 \\
\text { interven- } \\
\text { tional stud- } \\
\text { ies reported } \\
\text { in English } \\
\text { and } \\
\text { Spanish }\end{array}$ & $\begin{array}{l}\text { - Nutrition intervention } \\
\text { - Promotion of respon- } \\
\text { sive care and learn- } \\
\text { ing opportunities } \\
\text { - Conditional cash } \\
\text { transfer }\end{array}$ & Undernutrition & $\begin{array}{l}\text { Bayley Scales of Infant } \\
\text { Development (cogni- } \\
\text { tive/mental score, lan- } \\
\text { guage score, motor } \\
\text { score, socio-emotional } \\
\text { score) anytime } \\
\text { between } 0 \text { and } 60 \\
\text { months }\end{array}$ & $\begin{array}{l}\text { The effect size of promoting responsive- } \\
\text { ness on HAZ was not significant; } \\
\text { however, its pooled effect sizes on } \\
\text { cognitive, language and motor score } \\
\text { were } \\
4-5 \text { times larger than those of nutri- } \\
\text { tional supplementation }\end{array}$ & $\begin{array}{l}\text { - Social emotional } \\
\text { score } \\
\text { - Cognitive score } \\
\text { - Motor score }\end{array}$ & Good \\
\hline 2 & $\begin{array}{l}\text { Kristjansson E, } \\
2015^{(30)}\end{array}$ & $\begin{array}{l}\text { Low- to high- } \\
\text { income } \\
\text { countries }\end{array}$ & $\begin{array}{l}5400 \text { children aged } \\
3-60 \text { months }\end{array}$ & $\begin{array}{l}\text { Cochrane } \\
\text { review and } \\
\text { meta-analy- } \\
\text { sis from } 32 \\
\text { studies }\end{array}$ & $\begin{array}{l}\text { Supplementary feeding } \\
\text { interventions, alone } \\
\text { or with co-interven- } \\
\text { tion }\end{array}$ & Undernutrition & $\begin{array}{l}\text { Psychomotor } \\
\text { Development Index } \\
\text { Mental Development } \\
\text { Index }\end{array}$ & $\begin{array}{l}\text { Two studies showed moderate positive } \\
\text { effects of feeding on psychomotor } \\
\text { development }\end{array}$ & $\begin{array}{l}\text { Psychomotor develop- } \\
\text { ment }\end{array}$ & Good \\
\hline 3 & Ip P, 2017 & $\begin{array}{l}\text { Low- to middle- } \\
\text { income studies }\end{array}$ & $\begin{array}{l}\text { Children aged } \leq 8 \\
\text { years }\end{array}$ & $\begin{array}{l}\text { Meta-analysis } \\
\text { from } 33 \\
\text { intervention } \\
\text { studies }\end{array}$ & • Nutrition intervention & Undernutrition & Cognitive development & $\begin{array}{l}\text { Childhood nutritional supplementation } \\
\text { could improve children's cognitive } \\
\text { development }(d 0.08 ; 95 \% \mathrm{Cl} 0.03 \text {, } \\
0.13 \text { ) and those with } \geq 5 \text { nutrients was } \\
\text { particularly beneficial ( } d 0.15 ; 95 \% \\
\mathrm{Cl} 0.08,0.22) \text {. }\end{array}$ & $\begin{array}{l}\text { Cognitive develop- } \\
\text { ment }\end{array}$ & Poor \\
\hline 4 & $\begin{array}{l}\text { Yousafzai AK, } \\
2014^{(23)}\end{array}$ & Pakistan & $\begin{array}{l}1486 \text { infants } \\
\text { aged } \leq 2 \cdot 5 \\
\text { months at enrol- } \\
\text { ment }\end{array}$ & $\begin{array}{l}\text { Community- } \\
\text { based clus- } \\
\text { ter rando- } \\
\text { mised trial } \\
\text { until the } \\
\text { child was } \\
24 \text { months } \\
\text { of age }\end{array}$ & $\begin{array}{l}80 \text { clusters of children } \\
\text { to receive the follow- } \\
\text { ing intervention for } \\
\text { - Control: Routine } \\
\text { health and nutrition } \\
\text { services } \\
\text { - Nutrition education } \\
\text { and multiple micro- } \\
\text { nutrient } \\
\text { powders } \\
\text { - Responsive stimula- } \\
\text { tion (responsive } \\
\text { stimulation) } \\
\text { - Combination of both } \\
\text { responsive stimula- } \\
\text { tion and nutrition } \\
\text { intervention }\end{array}$ & $\begin{array}{l}\text { Generally } \\
\text { healthy chil- } \\
\text { dren }\end{array}$ & $\begin{array}{l}\text { Bayley Scales of Infant } \\
\text { and Toddler } \\
\text { Development, Third } \\
\text { Edition at } 12 \text { and } 24 \\
\text { months of age }\end{array}$ & $\begin{array}{l}\text { - Children who received responsive stimu- } \\
\text { lation had significantly higher develop- } \\
\text { ment scores on the cognitive, } \\
\text { language, and motor scales at } 12 \text { and } \\
24 \text { months of age, and on the social- } \\
\text { emotional scale at } 12 \text { months of age, } \\
\text { than control } \\
\text { - Children who received enhanced nutri- } \\
\text { tion had significantly higher develop- } \\
\text { ment scores on the cognitive, language } \\
\text { and social-emotional scales at } 12 \\
\text { months of age than control, but at } 24 \\
\text { months of age only the language } \\
\text { scores remained significantly higher } \\
\text { - No additive benefits when responsive } \\
\text { stimulation was combined with nutrition } \\
\text { interventions } \\
\text { - Children exposed to enhanced nutrition } \\
\text { had significantly better HAZ at } 6 \text { and } 18 \\
\text { months than did children not exposed to } \\
\text { enhanced nutrition } \\
\text { - Treatment effect on cognition, language } \\
\text { and motor development at } 24 \text { months } \\
\text { were moderate to large for responsive } \\
\text { simulation and were low to moderate } \\
\text { for the enhanced nutrition group }\end{array}$ & $\begin{array}{l}\text { - Cognitive scales } \\
\text { - Language scales } \\
\text { - Motor scales }\end{array}$ & Good \\
\hline
\end{tabular}


Table 2. (Continued)

\begin{tabular}{|c|c|c|c|c|c|c|c|c|c|c|}
\hline No. & $\begin{array}{l}\text { First author, year } \\
\text { of publication }\end{array}$ & Country of study & $\begin{array}{l}\text { Sample size and } \\
\text { age at enrolment }\end{array}$ & $\begin{array}{l}\text { Type of } \\
\text { studies }\end{array}$ & Type of intervention & $\begin{array}{l}\text { Nutritional } \\
\text { status of the } \\
\text { study popula- } \\
\text { tion }\end{array}$ & $\begin{array}{l}\text { Developmental tools } \\
\text { and age at assessment }\end{array}$ & Key findings & $\begin{array}{l}\text { Affected cognitive } \\
\text { and neurodevelop- } \\
\text { mental aspects by } \\
\text { suboptimal growth }\end{array}$ & $\begin{array}{l}\text { Study } \\
\text { quality }\end{array}$ \\
\hline 5 & $\begin{array}{l}\text { Rosado JL, } \\
2011^{(22)}\end{array}$ & Mexico & $\begin{array}{l}422 \text { children aged } \\
1-24 \text { months }\end{array}$ & $\begin{array}{l}\text { Randomised, } \\
\text { placebo- } \\
\text { controlled } \\
\text { longitudinal } \\
\text { trial for } 6 \\
\text { months }\end{array}$ & $\begin{array}{l}\text { - Oral food supplement } \\
\text { (OFS) with higher } \\
\text { content of carbohy- } \\
\text { drates and micronu- } \\
\text { trients, including Fe } \\
\text { and Zn, than did the } \\
\text { PM } \\
\text { - Powdered milk (PM) } \\
\text { - Placebo (PL) }\end{array}$ & $\begin{array}{l}\text { Healthy popu- } \\
\text { lation }\end{array}$ & $\begin{array}{l}\text { Bayley Scale of Infant } \\
\text { Development Test for } \\
\text { cognitive and motor } \\
\text { functions after } 6 \\
\text { months from enrolment }\end{array}$ & $\begin{array}{l}\text { - Daily supplementation of } \\
12-24 \text {-month-old children with OFS } \\
\text { has no additional benefits in growth, } \\
\text { anaemia, morbidity or cognitive per- } \\
\text { formance } \\
\text { - No significant effect of treatment was } \\
\text { found after } 6 \text { months in the prevalence } \\
\text { of stunting after adjusting for initial val- } \\
\text { ues in the OFS or PM group compared } \\
\text { with PL }\end{array}$ & None & Good \\
\hline 6 & Kvestad I, 2015 & India & $\begin{array}{l}422 \text { children aged } \\
6-30 \text { months }\end{array}$ & $\begin{array}{l}\text { Randomised, } \\
\text { double- } \\
\text { blind, } \\
\text { placebo-con- } \\
\text { trolled trial } \\
\text { for } 6 \\
\text { months }\end{array}$ & $\begin{array}{l}\text { Placebo group } \\
\text { versus } \\
\text { intervention group (vita- } \\
\text { min B12 only, folic } \\
\text { acid only, and vita- } \\
\text { min B12 and folic } \\
\text { acid) with (1:1:1:1 } \\
\text { ratio) }\end{array}$ & Undernutrition & $\begin{array}{l}\text { Ages and Stages } \\
\text { Questionnaire 3rd ed. } \\
\text { after } 6 \text { months from } \\
\text { enrolment }\end{array}$ & $\begin{array}{l}\text { - Children who received both vitamin } \\
\text { B12 and folic acid had } 0.45(95 \% \mathrm{Cl} \\
0.19,0.73) \text { and } 0.28 \text { (95 \% } \mathrm{Cl} 0.02 \text {, } \\
0.54) \text { higher } \\
\text { SD-units in the domains of gross } \\
\text { motor and problem-solving function- } \\
\text { ing, respectively. } \\
\text { - Effect was highest in stunted children, } \\
\text { those with high plasma homocysteine } \\
\text { (>10 } 10 \mathrm{~mol} / \mathrm{L}) \text { or in those who were } \\
\text { younger than } 24 \text { months at end study } \\
\text { - Timing of nutritional influence is of sig- } \\
\text { nificance for the outcome of neurode- } \\
\text { velopment }\end{array}$ & $\begin{array}{l}\text { - Gross motor } \\
\text { - Problem-solving } \\
\text { skills }\end{array}$ & Good \\
\hline 7 & $\begin{array}{l}\text { Larson LM, } \\
\quad 2017^{(44)}\end{array}$ & $\begin{array}{l}\text { Low- and } \\
\text { middle-income } \\
\text { countries }\end{array}$ & $\begin{array}{l}5400 \text { children aged } \\
0-24 \text { months }\end{array}$ & $\begin{array}{l}\text { Systematic } \\
\text { review on } \\
\text { interven- } \\
\text { tional rand- } \\
\text { omised } \\
\text { studies }\end{array}$ & $\begin{array}{l}\text { Systematic review on } \\
\text { nutrition intervention } \\
\text { pre- and postnatal }\end{array}$ & $\begin{array}{l}\text { Undernutrition } \\
\text { and healthy } \\
\text { population }\end{array}$ & $\begin{array}{l}\text { Assessment during the } \\
\text { first } 48 \text { months of life: } \\
\text { - BSID-I, -II or -III - } \\
\text { Mental Scale } \\
\text { - Griffiths Mental } \\
\text { Developmental Scale } \\
\text { - Fagan Test of Infant } \\
\text { Intelligence }\end{array}$ & $\begin{array}{l}\text { - Motor development, but not growth sta- } \\
\text { tus, effect sizes were significantly } \\
\text { associated with mental development in } \\
\text { postnatal } \\
\text { interventions } \\
\text { - Nutrition interventions had small effects } \\
\text { on mental development } \\
\text { - Baseline and endline HAZ was not a } \\
\text { significant predictor of postnatal effect } \\
\text { size, and baseline maternal BMI was } \\
\text { not a significant predictor of prenatal } \\
\text { effect size }\end{array}$ & Motor development & Good \\
\hline 8 & $\begin{array}{l}\text { Walker SP, } \\
2010^{(28)}\end{array}$ & Jamaica & $\begin{array}{l}\text { Normal birth weight } \\
\text { newborns }(n \\
=73) \text { compared } \\
\text { with low-birth } \\
\text { weight, term- } \\
\text { born newborns } \\
\text { (LBWT) }(n=99)\end{array}$ & $\begin{array}{l}\text { Randomised } \\
\text { control } \\
\text { study }\end{array}$ & $\begin{array}{l}\text { Psychosocial stimula- } \\
\text { tion assessed with } \\
\text { the Middle Childhood } \\
\text { Home Observation } \\
\text { for the Measurement } \\
\text { of the Environment } \\
\text { (MC-HOME) }\end{array}$ & $\begin{array}{l}\text { Low birth } \\
\text { weight and } \\
\text { normo-birth } \\
\text { weight }\end{array}$ & $\begin{array}{l}\text { At } 72 \text { months: } \\
\text { - Wechsler Preschool } \\
\text { and Primary Scale of } \\
\text { Intelligence, 3rd } \\
\text { edition (WPPSI-III) } \\
\text { - Digit span forwards test } \\
\text { and visual-spatial } \\
\text { memory with the Corsi } \\
\text { blocks test } \\
\text { - Test of Everyday } \\
\text { Attention for Children } \\
\text { - Early Reading } \\
\text { Assessment } \\
\text { - Strengths and } \\
\text { Difficulties } \\
\text { Questionnaire (SDQ) }\end{array}$ & $\begin{array}{l}\text { - Compared with normal-birth-weight } \\
\text { children, LBWT had poorer selective } \\
\text { attention and visual-spatial memory, } \\
\text { but there were no differences in IQ, } \\
\text { language or behaviour } \\
\text { - LBWT who received stimulation interven- } \\
\text { tion had higher IQ score and fewer } \\
\text { behavioural problems as compared } \\
\text { with the control group }\end{array}$ & $\begin{array}{l}\text { - Selective attention } \\
\text { - Visual-spatial } \\
\text { memory }\end{array}$ & Poor \\
\hline
\end{tabular}


Table 3. Factors influencing suboptimal growth in neurodevelopment and cognition

\begin{tabular}{|c|c|c|c|}
\hline Maternal factors & Paternal factors & Child's characteristics and health condition & Socio-economic conditions \\
\hline \multicolumn{4}{|l|}{ Undernourished and normo-nourished children } \\
\hline Schooling (<tertiary education) & Schooling (<tertiary education) & Sex (mainly boy - conflicting evidence) & $\begin{array}{l}\text { Poor sanitation (not flushing the toilet } \\
\text { during childhood) }\end{array}$ \\
\hline Lower nutrition knowledge & Non-employment at child's birth & Birth weight deficit & Poor water quality \\
\hline Teenage at delivery & Teenage at delivery & Birth length deficit & $\begin{array}{l}\text { Wealth/household income ( } \leq 1 \text { monthly } \\
\text { minimum wage) }\end{array}$ \\
\hline Low BMI $\left(18.10 \mathrm{~kg} / \mathrm{m}^{2}\right)$ & Height deficit & Birth head circumference deficit & No consumption of dinner \\
\hline Height deficit/decreased stature & Lower nutrition knowledge & $\begin{array}{l}\text { Growth trajectories (head circumference length, } \\
\text { height and weight deficit) }\end{array}$ & $\begin{array}{l}\text { Housing condition ( } \geq 3 \text { persons per } \\
\text { room) }\end{array}$ \\
\hline $\begin{array}{l}\text { Non-employment between pregnancy and the } \\
\text { child's first } 12 \text { months of life }\end{array}$ & Perceiving less responsibility in the feeding task & Prematurity (<37 weeks) & Low HOME environment \\
\hline Perceiving less responsibility in the feeding task & $\begin{array}{l}\text { Being less restrictive towards access to unhealthy } \\
\text { foods }\end{array}$ & $\begin{array}{l}\text { Exposure and duration of breastfeeding }(<1 \\
\text { month) }\end{array}$ & $\begin{array}{l}\text { Play time with a caregiver }(<1 \mathrm{~h} \text { per } \\
\text { day) }\end{array}$ \\
\hline $\begin{array}{l}\text { Being less restrictive towards access to unhealthy } \\
\text { foods }\end{array}$ & $\begin{array}{l}\text { Engagement in activities with the child (0-2 activities } \\
\text { versus } 7 \text { activities per week) }\end{array}$ & Duration of exclusive breastfeeding ( $<1$ month) & Having no toys or books \\
\hline $\begin{array}{l}\text { Presence of mental condition during the child's } \\
\text { first year of life }\end{array}$ & & No postnatal motor development interventions & $\begin{array}{l}\text { Being born within less than } 24 \text { months } \\
\text { after the first baby }\end{array}$ \\
\hline $\begin{array}{l}\text { Level of physical activity before and during preg- } \\
\text { nancy (inactive) }\end{array}$ & & Low haemoglobin level at 9 months $(<11.5 \mathrm{~g} / \mathrm{dL})$ & Number of siblings $(\geq 3)$ \\
\hline Ethnicity (mainly non-white/African) & & Ethnicity (mainly non-white/African) & Non-availability of health facilities \\
\hline Smoking during pregnancy & & & No childcare during the first year of life \\
\hline \multicolumn{4}{|l|}{ Unintended pregnancy } \\
\hline \multicolumn{4}{|l|}{ Having no partner } \\
\hline \multicolumn{4}{|l|}{ Number of pre-natal visits $(<6)$} \\
\hline \multicolumn{4}{|l|}{ No hospitalisation during pregnancy } \\
\hline \multirow{2}{*}{\multicolumn{4}{|c|}{$\begin{array}{l}\text { Vaginal delivery } \\
\text { Overweight and obese children }\end{array}$}} \\
\hline & & & \\
\hline- & - & Low socio-economic status & - \\
\hline
\end{tabular}

HOME, Home Observation for Measurement of the Environment 


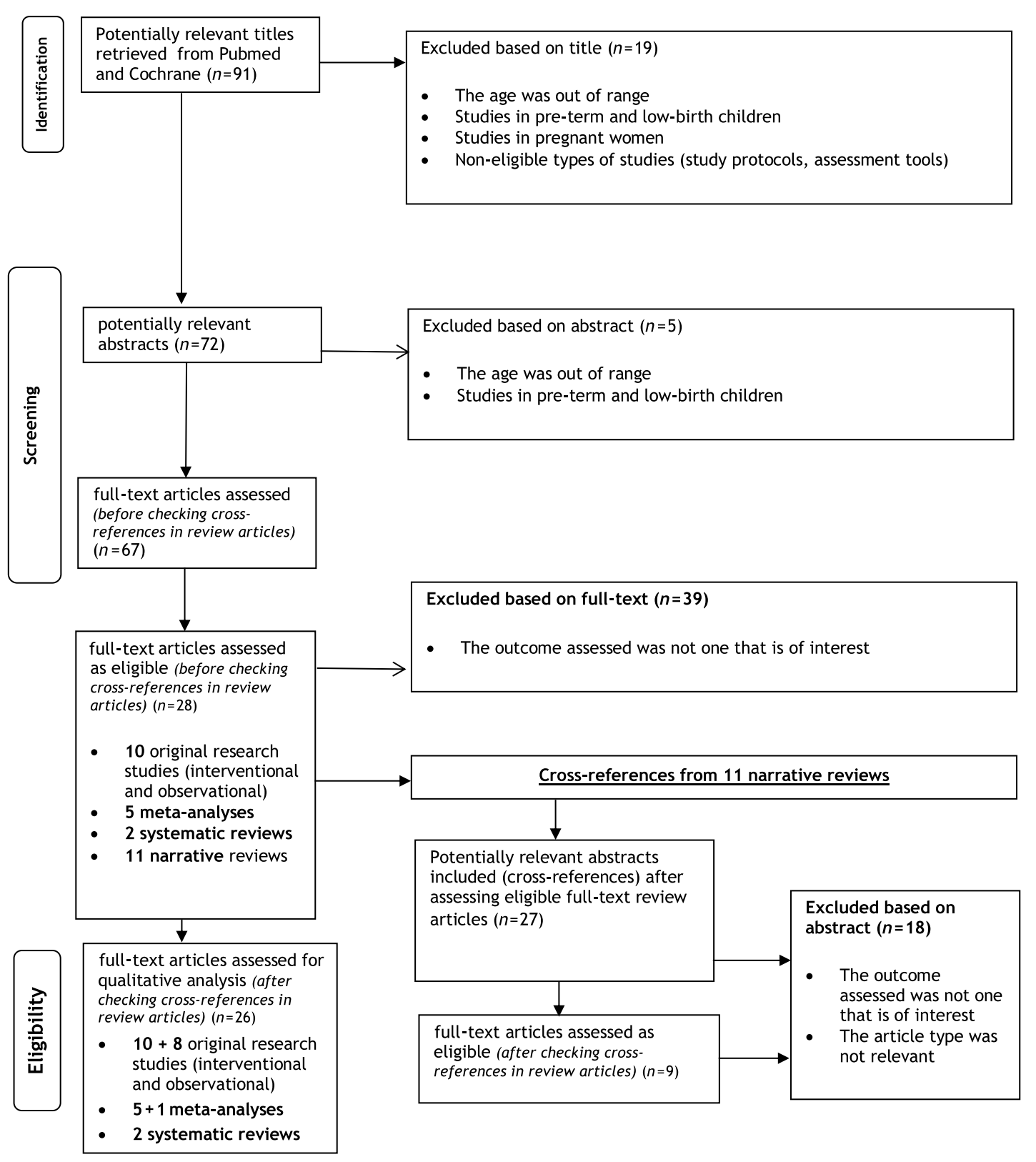

Fig. 1. PRISMA 2009 flowchart diagram.

gain (or less loss) in HAZ after the age of 6 months was positively associated with cognitive function at 11 years of age. In addition, changes in HAZ from 6 to 24 months and changes in HAZ from 24 months to 11 years were also positively associated with cognitive ability at 11 years in the same study ${ }^{(11)}$. These outcomes are similar to the results among children in Bangladesh. In this study, cognitive impairment was associated with poverty, birth condition and postnatal growth that started at 7 months of age and worsened up to 64 months when it became even more substantial ${ }^{(12)}$. The impact of the timing of stunting is still unclear. One study reported that stunting in the second year of life is more harmful than stunting before 12 months of age ${ }^{(13)}$. Another study reported that higher HAZ at 4-6 years of age among healthy Malaysian children significantly contributed to higher cognitive function after controlling for socio-demographic background, parent's nutrition knowledge and dinner consumption $(\mathrm{DR} 2=0.009, \mathrm{DF}=18.605, p<0.001)^{(14)}$.

Evidence for the impact of underweight and stunting on the development of emotional skills is largely variable across the identified studies. Two meta-analyses that described data from lower- and middle-income countries showed the limited influence of undernutrition on a child's attachment, emotionality, social competence and temperament ${ }^{(6,8)}$. However, in a study among Bangladeshi children aged 6-24 months comparing undernourished $(n=212)$ and better-nourished $(n=108)$, it was reported that severely underweight children (WAZ $<-2$ ) were less sociable, less attentive, and more fearful and had more negative emotional traits than normo-nourished children. The 


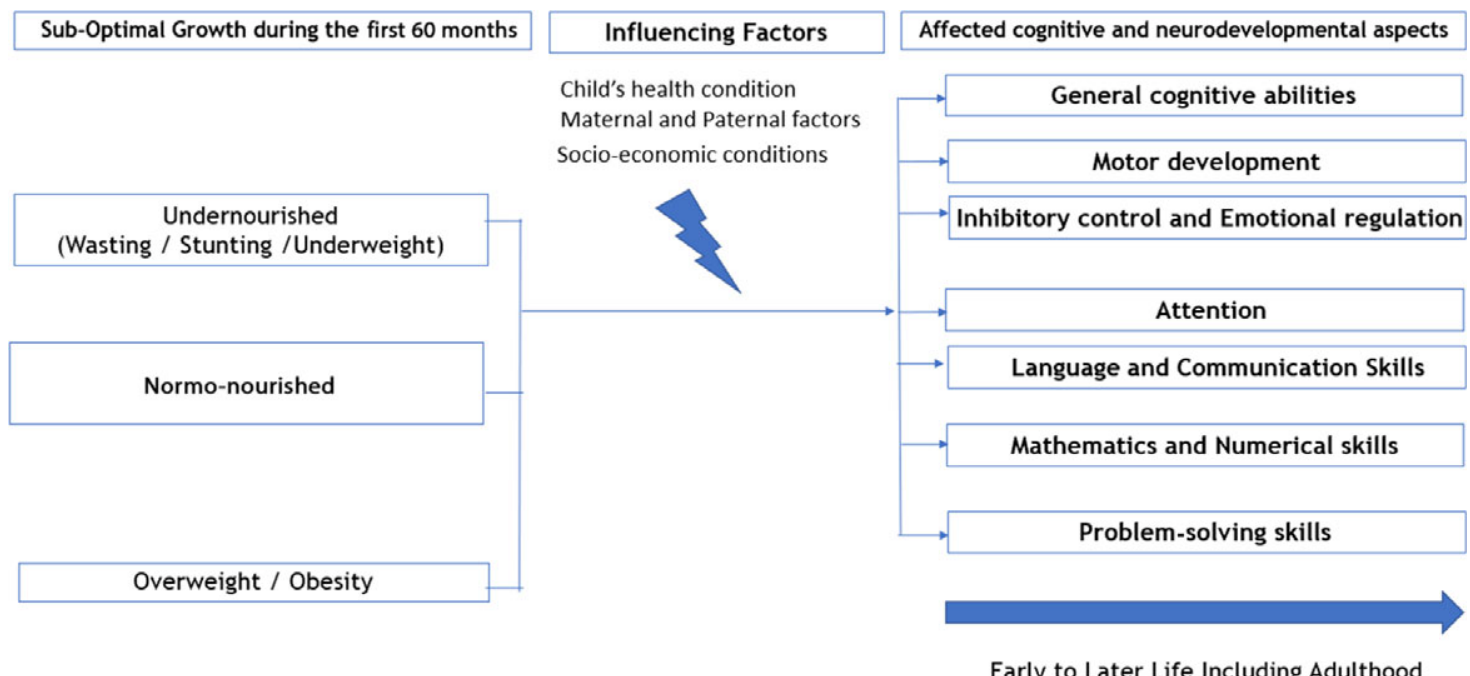

Fig. 2. Impact of suboptimal growth in early childhood on developmental-related aspects.

effect size of these temperament differences, which were assessed using validated interviewer-administered questionnaire to parents, were small to moderate ${ }^{(15)}$. There is evidence, therefore, that underweight and stunting impact social skill development. Low HAZ and WAZ correlated strongly with children having less schooling or needing a longer time to complete schooling $^{(16)}$, and in later life, with lower income and living in a less conducive environment, such as having a higher number of pregnancies and having the first child at a younger age ${ }^{(17)}$.

\section{Improvements in linear growth and potential window of opportunity for neurodevelopment and cognitive abilities recovery}

This review further strengthens the importance of the first 1000 days of life as a window of opportunity for rescuing neurocognitive deficits. It was reported that HAZ velocity between 6 and 24 months among undernourished children is positively associated with cognitive function ${ }^{(11)}$ and that every 1 -unit increase in HAZ before the age of 24 months is associated with a higher increment in motor, communication and cognitive ability scores at 26-80 months of age compared with a 1-unit increase in HAZ after 24 months of age ${ }^{(6,16)}$.

Data from the Young Lives cohort in Ethiopia, India, Peru, Cambodia and Vietnam indicated that children who were in the recovered group (stunted at 12 months of age but not stunted at 8 years of age) had better academic achievement (maths scores, receptive vocabulary scores, and reading comprehension scores) than those who stayed persistently stunted ${ }^{(16)}$. However, their academic achievement was still lower than those who had never been stunted. This finding is consistent with the finding from another cohort in Malawi, which reported that those who had recovered from stunting had better academic achievements at 11 years of age than those who were persistently stunted from age 4 to 8 years ${ }^{(18)}$. Based on the above, it appears likely that the period in which neurocognitive deficits can recover following appropriate interventions goes beyond the age of 2 years, up until 8 years of age, which is in line with prefrontal cortex development and ongoing synaptogenesis at that age ${ }^{(5)}$. Interestingly, another study reported that children who were stunted at 24 months of age and experienced catch-up growth often did worse on cognitive tests at 4-5 years of age than children who were never stunted and did almost as poorly as children who had remained stunted ${ }^{(19)}$. This finding appears to contradict previously stated findings from the Young Lives and Malawi cohorts. It is important to note, however, that the exact definition of 'catch-up' plays a critical role in this observation. Recently, five definitions were formulated for 'catch-up growth', ranging from lenient to strict: I: increase in HAZ; II: recovery from stunting (HAZ at 5 years $>-2$ ); III: increase in height-for-age difference; IV: recovery from stunting and increase in height-for-age difference; and V: recovery from stunting with a stricter cut-off point, where HAZ falls within 'normal' range (HAZ at 5 years $>-1)^{(20)}$. The observation reported above is based on definitions I-IV of 'catch-up'. Thus, the term 'catch-up' should be carefully defined when determining the optimal goal for catch-up growth to recover neurodevelopment and, consequently, cognitive ability in later life.

\section{The importance of interventions in improving cognitive outcomes among undernourished children}

Seven publications assessed the effectiveness of intervention studies in improving cognitive outcomes among undernourished children. The publications covered three randomised controlled trials, two systematic reviews and two meta-analyses. The overall quality of these studies was judged as good when considering the risk for bias (Table 2).

Stunted children with high levels of plasma homocysteine and those who were younger than 24 months at the end of the follow-up period showed the largest improvement in gross motor function and problem-solving skills after being supplemented with vitamin B12 on a daily basis ${ }^{(21)}$. In contrast, the effect of the vitamin/nutrition intervention on recovering or maintaining normal neurodevelopment and cognitive deficits in normo-nourished children remained inconclusive ${ }^{(7,21,22)}$. No 
benefits in growth or cognitive performance were reported among 422 healthy children aged 12-24 months receiving daily multi-micronutrient supplementation (iron and zinc) ${ }^{(22)}$. This finding is in contrast with an intervention trial in which multiple micronutrients were supplemented (vitamins B12, A, C, folic acid and iron), with and without responsive stimulation, for approximately 20 months among 1489 mother-child pairs in Pakistan. In this study, children who were exposed to the enhanced nutrition intervention had, regardless of their nutrition status, significantly higher developmental scores (cognitive, language and social-emotional scales) at 12 months than the control group ${ }^{(23)}$. Another group of children who received responsive stimulation had significantly higher developmental scores on the cognitive, language and motor scales at 12 and 24 months of age and on the social emotional scale at 12 months of age than the control group. In comparison, the treatment effect on cognition, language and motor development at 24 months was moderate to large for responsive simulation and was low to moderate for the enhanced nutrition group ${ }^{(23)}$. This study also found that linear growth in children exposed to enhanced nutrition was significantly greater at 6 and 18 months compared with those not exposed to enhanced nutrition ${ }^{(22)}$.

A recent systematic review and meta-analysis that assessed the impact of nutrition with or without stimulation interventions concluded that parent-led responsive learning, more than nutrition supplementation, appeared to have a stronger influence on the neurodevelopment of children aged 0-60 months ${ }^{(7)}$. This systematic review, which was based on seventy-five retrieved studies from various databases, reported that the pooled effect of these supplementations is one-fourth smaller than the pooled effect size of responsive care and learning opportunities on cognitive, language and motor scales. The review also suggested that postnatal multiple nutrient supplementation can improve linear growth in children and, to a smaller extent, cognitive development and socio-temperament, while interventions promoting responsive care and learning opportunities affect only cognitive development but not linear growth ${ }^{(7)}$.

\section{The impact of overweight/obesity on neurodevelopment and cognition}

Only one cross-sectional study and two systematic reviews that were retrieved described the impact of overweight/obesity on cognitive neurodevelopmental aspects ${ }^{(24-26)}$ (Table 1). All studies seemed to point towards a more limited impact of overweight/obesity on aspects of neurodevelopment and cognition in the studied affluent populations in contrast with those describing the impact of undernutrition. All retrieved literature demonstrated that attention, executive control (inhibitory control, working memory, reward sensitivity, and impulsivity) and gross motor skills were the most affected areas.

A study among overweight Italian children showed poorer gross motor skills in overweight children than their normalweight peers ${ }^{(24)}$, while other systematic reviews reported poorer executive control and more difficulty with inhibition in overweight children than in healthy weight children ${ }^{(25,26)}$. There is a suggestion of directionality in the association between obesity and executive function. Children with lower executive function are speculated to have lower self-regulation of energy intake and decreased participation in physical activity, which leads to or potentiates overweight or obesity. It was also reported that low executive function stimulates excess adiposity, leading to exacerbating decrements in executive function in childhood ${ }^{(27)}$. On the other hand, various studies have shown that child obesity or early-life adiposity reduces executive function via several biological mechanisms. Pro-inflammatory cytokines produced by adipose tissue can stimulate inflammatory pathways in all age categories, leading to cognitive development deficits, and dysregulation of appetite-regulating hormones could further harm cognitive skills ${ }^{(26)}$. Although there is limited affected cognitive neurodevelopmental areas reported in these affluent populations, the effect may be more diverse and pronounced in lessaffluent societies. In conclusion, poor gross motor skills, which are correlated with less physical activity ${ }^{(25)}$ and reduced attention $\operatorname{span}^{(25,26)}$, should be taken into consideration when designing intervention programs for these overweight/obese children.

\section{Factors influencing the impact of suboptimal growth on neurodevelopment and cognition}

From the literature, there seem to be similarities in influencing factors between undernutrition and overweight/obesity on cognitive neurodevelopment, but far more research is needed to further understand the latter. These factors can be classified into maternal and paternal factors, child characteristics and health conditions, and socio-economic factors (Table 3).

Several pregnancy-related maternal parameters, such as maternal weight gain and smoking, have been consistently reported to increase the impact of suboptimal growth on cognitive neurodevelopment in addition to infant condition at birth, such as low birth weight and short length at birth, suggesting the importance of prenatal nutrition and maternal health for later life $\mathrm{e}^{(12,15,19,28,29)}$.

The influence of sex on suboptimal growth and cognitive neurodevelopment appeared conflicting in the literature; normo-nourished and undernourished girls had slightly higher intelligence scores ${ }^{(10)}$, social maturity ${ }^{(19)}$, and manageability ${ }^{(15)}$ than boys with the same nutritional status. In contrast, another study found that normo-nourished boys tended to have higher intelligence score as compared with normo-nourished girls ${ }^{(14)}$.

Particularly among undernourished children, it appeared that parents with a low level of education and nutritional knowledge, those who perceived less responsibility in the parenting task, or those who were less restrictive towards access to unhealthy foods had children who experienced suboptimal growth with resultant impaired cognitive development ${ }^{(6,14)}$.

Diet quality was demonstrated to be a positive predictor of improved cognitive outcomes in these children ${ }^{(6,14)}$. For instance, food fortified with calcium, iron, zinc, vitamin B2 and protein was associated with improved cognitive outcomes because of the probable role of these nutrients in early brain development ${ }^{(7,23)}$. In contrast, a lack of dietary protein may delay or inhibit brain and cognitive development ${ }^{(6,30)}$. On that note, recent evidence proposed supplementation of animal protein, in particular cow's milk, as an essential component of a child's diet to prevent undernutrition and to improve cognition ${ }^{(31-33)}$. 
Low socio-economic status was reported to be correlated with the impact on neurodevelopment and cognition among undernourished and overweight/obese children in several studies $^{(6,10,23,28,29,34,35)}$. The Home Observation for Measurement of the Environment (HOME) parameter was used as a proxy indicator of socio-economic status. It was developed to measure responsiveness and stimulation in the home environment and parental behaviours. HOME consisted of questions, among others, on homeownership and possession of books and toys. The tool needs to be validated to accommodate sociocultural differences ${ }^{(29,36)}$

\section{Limitations and strengths of the review}

First, chronic illnesses and hospital malnutrition were excluded, although they could have a similar impact on overall developmental aspects. A second limitation is that the HOME instrument as an indicator of socio-economic status must be adjusted to various sociocultural conditions in each group or country. In addition, the statistical significance of pooled effects of all studies combined was not calculated, as this review is of scoping nature and not a systematic review. Furthermore, the search strategy may have missed some relevant papers because of the search scope that limited peer-reviewed research papers to those published in the past decade only. To overcome this issue, reviewing reports outside the peer-reviewed literature (i.e. grey literature) should be done in the future.

One of the strengths of this scoping review is that it expeditiously provides comprehensive and updated scientific evidence for healthcare professionals and policymakers. The synthesised evidence may allow key stakeholders to plan and to execute necessary intervention programs for preventing and managing suboptimal growth, with a focus on achieving optimal cognitive potential in young children. In addition, this scoping review was conducted using specific methodological approaches that would allow more accuracy in literature screening with less bias. A cross-referencing of eligible studies from retrieved narrative reviews enhanced the completeness of the evidence and enabled the retrieval of information on not only the populations that experience undernutrition and overweight/obesity but also the general population that identified children with faltering growth. It also enabled the retrieval of studies from various geographical areas (low- to high-income studies) as well as studies on various developmental deficits. Emphasis had been hitherto placed on the impact of malnutrition in children on morbidity and mortality; in contrast, this review points to the quality of life that may be affected by suboptimal nutrition on neurodevelopment and cognition. From a public health perspective, prevention of childhood malnutrition must be addressed not only by economic empowerment but also through health promotion and education as well as policy.

\section{Consideration for future research and actions:}

1) More studies should be conducted on the impact of nutritional/behavioural interventions in overweight/obese children on neurodevelopment and cognition, especially in low-income countries, as the currently retrieved literature all came from high-income countries. Overweight/obesity in childhood is on the rise and can coexist with undernutrition within the same population, for example, as seen in India and Indonesia. Approximately, 39 and 7 million children under the age of 5 years are stunted in India and Indonesia,respectively, and more than 3.6 million children in the same age group areoverweight and obese in these two countries. This amounts to a total of around 50 million children who are at risk of suboptimal neurodevelopment and cognitivefunction $^{(3)}$. In addition to the negative consequences of undernutrition, an increase in childhood obesity prevalence can lead to adverse long-term consequences on human capital in these countries.

2) Intervention studies for both undernutrition and overweight/ obesity must assess the association between these nutritional statuses and cognitive neurodevelopment, as most interventions usually focus only on providing nutrients related to brain development and not for optimal growth and vice versa as also suggested by Prado et al. ${ }^{(7)}$. For example, several recently published studies and systematic reviews assessed the impact of animal-based food supplementation or milk interventions on either growth among stunted children or only cognitive neurodevelopment, but not on both aspects simultaneously ${ }^{(31,32)}$

3) Optimally, any program or intervention for growth monitoring in the community or hospital settings should engage a multidisciplinary team of health care professionals. Depending on the local conditions, such a team could consist of nutritionists, general practitioners, paediatricians and/or behavioural specialists ${ }^{(37)}$. Multidimensional approaches involving various relevant stakeholders need to address all issues simultaneously as the root causes are quite complex. These could include improving housing and sanitation levels, ensuring parental job security and adequate social welfare, improving parental education attainment and nutritional knowledge, and providing access to adequate nutrient intakes for catch-up growth among underweight or stunted children as well as to affordable healthy food for those who are overweight/obese especially those in the low socio-economic category. Actions taken should tackle both undernutrition and overweight/obesity concurrently - not just one or the other - to ensure successful and comprehensive outcomes in terms of childhood growth and cognitive neurodevelopment.

\section{Conclusion}

Undernutrition and overweight/obesity during the first 60 months of postnatal life affect the cognitive neurodevelopmental trajectories of children later in life. Weight and length/height need to be monitored even beyond 24 months of life to enable early recognition of growth retardation/deviations and to allow appropriate and timely interventions to address their negative neurodevelopmental and cognitive impacts. Given the Global Nutrition Targets for $2025^{(38)}$, intervention programs should leverage multidimensional approaches that address not only 
childhood undernutrition but also overweight/obesity and improve socio-economic conditions. Nutrition interventions for catch-up growth among undernourished children can potentially recover neurocognitive development up to the age of 8 years, particularly in those whose nutrition status have improved. These specific, targeted and simultaneous interventions may be key to safeguarding future human capital, especially in countries with a high prevalence of stunting, overweight/obesity or both.

\section{Declarations}

\section{Acknowledgements}

The authors thank Content Ed Net Singapore for providing editorial and medical writing assistance in retrieving relevant publications and the development of the first drafts of the manuscript.

\section{Funding}

FrieslandCampina supported the retrieval of relevant publications and the development of the early first manuscript drafts by Content Ed Singapore. None of the authors received an educational grant from FrieslandCampina to participate in the writing.

\section{Author contributions}

All authors were involved in setting the concept, objective and selection criteria for the review. All authors were also involved in the selection of eligible articles based on titles and abstracts. A simple grading of controlling study biases was then conducted by five authors (A.S., M.Y.J., P.B.K., L.M. and J.G.). Afterwards, L.M. and J.G. selected eligible articles based on full texts. All authors provided agreement on the final included articles. All authors participated in data extraction, synthesis and interpretation. All authors provided inputs and agreed on the final version of the manuscript.

\section{Competing interests}

Verena Tan was an employee of FrieslandCampina at the time of the manuscript's development. Leilani Muhardi and Jan Geurts were employees of FrieslandCampina at the time of the manuscript's development, submission and revision.

\section{Consent for publication}

Not applicable.

\section{Ethics approval and consent to participate}

Not applicable.

\section{References}

1. Global Nutrition Report: Action on equity to end malnutrition. Development Initiatives 2020.

2. Popkin BM, Corvalan C \& Grummer-Strawn LM (2020) Dynamics of the double burden of malnutrition and the changing nutrition reality. Lancet 395(10217), 65-74.

3. Global Nutrition Report: Shining a light to spur action on nutrition. Development Initiatives. 2018.

4. de Onis MOA, Borghi E, Siyam A, Pinol A (2006) WHO Child Growth Standards. World Health Organization.

5. Grantham-McGregor S, Cheung YB, Cueto S, et al. (2007) Developmental potential in the first 5 years for children in developing countries. Lancet 369(9555), 60-70.

6. Sudfeld CR, McCoy DC, Danaei G, et al. (2015) Linear growth and child development in low-and middle-income countries: a meta-analysis. Pediatrics 135(5), e1266-75.

7. Prado EL, Larson LM, Cox K, et al. (2019) Do effects of early life interventions on linear growth correspond to effects on neurobehavioural development? A systematic review and metaanalysis. Lancet Glob Health 7(10), e1398-e1413.

8. Miller AC, Murray MB, Thomson DR, et al. (2016) How consistent are associations between stunting and child development? Evidence from a meta-analysis of associations between stunting and multidimensional child development in fifteen low- and middle-income countries. Public Health Nutr 19 , 1339-47.

9. Munn Z, Peters MDJ, Stern C, et al. (2018) Systematic review or scoping review? Guidance for authors when choosing between a systematic or scoping review approach. BMC Med Res Methodol 18(1), 143.

10. Camargo-Figuera FA, Barros AJ, Santos IS, et al. (2014) Early life determinants of low IQ at age 6 in children from the 2004 Pelotas Birth Cohort: a predictive approach. BMC Pediatr 14, 308.

11. Cheung YB \& Ashorn P (2010) Continuation of linear growth failure and its association with cognitive ability are not dependent on initial length-for-age: a longitudinal study from 6 months to 11 years of age. Acta Paediatr 99(11), 1719-23.

12. Hamadani JD, Tofail F, Huda SN, et al. (2014) Cognitive deficit and poverty in the first 5 years of childhood in Bangladesh. Pediatrics 134(4), e1001-8.

13. Mendez MA \& Adair LS (1999) Severity and timing of stunting in the first two years of life affect performance on cognitive tests in late childhood. J Nutr 129(8), 1555-62.

14. Mohd Nasir MT, Norimah AK, Hazizi AS, et al. (2012) Child feeding practices, food habits, anthropometric indicators and cognitive performance among preschoolers in Peninsular Malaysia. Appetite 58(2), 525-30.

15. Baker-Henningham H, Hamadani JD, Huda SN, et al. (2009) Undernourished children have different temperaments than better-nourished children in rural Bangladesh. J Nutr 139(9), 1765-71.

16. Crookston BT, Schott W, Cueto S, et al. (2013) Postinfancy growth, schooling, and cognitive achievement: Young Lives. Am J Clin Nutr 98(6), 1555-63.

17. Hoddinott J, Behrman JR, Maluccio JA, et al. (2013) Adult consequences of growth failure in early childhood. Am J Clin Nutr 98(5), 1170-8.

18. Sunny BS, DeStavola B, Dube A, et al. (2018) Does early linear growth failure influence later school performance? A cohort study in Karonga district, northern Malawi. PLoS One 13(11), e0200380.

19. Casale D, Desmond C \& Richter L (2014) The association between stunting and psychosocial development among preschool children: a study using the South African Birth to Twenty cohort data. Child Care Health Dev 40(6):, 900-10. 
20. Desmond C \& Casale D (2017) Catch-up growth in stunted children: definitions and predictors. PLoS One 12(12), e0189135.

21. Kvestad I, Taneja S, Kumar T, et al. (2015) Vitamin B12 and folic acid improve gross motor and problem-solving skills in young North Indian children: a randomized placebocontrolled trial. PLoS One 10(6), e0129915.

22. RosadoJL, Lopez P, Garcia OP, et al. (2011) Effectiveness of the nutritional supplement used in the Mexican Oportunidades programme on growth, anaemia, morbidity and cognitive development in children aged 12-24 months. Public Health Nutr 14(5), 931-7.

23. Yousafzai AK, Rasheed MA, Rizvi A, et al. (2014) Effect of integrated responsive stimulation and nutrition interventions in the Lady Health Worker programme in Pakistan on child development, growth, and bealth outcomes: a cluster-randomised factorial effectiveness trial. Lancet 384(9950), 1282-93.

24. Morano M, Colella D \& Caroli M (2011) Gross motor skill performance in a sample of overweight and non-overweight preschool children. Int J Pediatr Obes, 6 Suppl 2, 42-6.

25. Liang J, Matheson BE, Kaye WH, et al. (2014) Neurocognitive correlates of obesity and obesity-related behaviors in children and adolescents. Int J Obes (Lond) 38(4), 494-506.

26. Reinert KR, Po'e EK \& Barkin SL (2013) The relationship between executive function and obesity in children and adolescents: a systematic literature review. J Obes 2013, 820956.

27. Hayes JF, Eichen DM, Barch DM, et al. (2018) Executive function in childhood obesity: Promising intervention strategies to optimize treatment outcomes. Appetite 124, 10-23.

28. Walker SP, Chang SM, Younger N, et al. (2010) The effect of psychosocial stimulation on cognition and behaviour at 6 years in a cohort of term, low-birthweight Jamaican children. Dev Med Child Neurol 52(7), e148-54.

29. Nguyen $\mathrm{PH}$, DiGirolamo AM, Gonzalez-Casanova I, et al. (2018) Influences of early child nutritional status and bome learning environment on child development in Vietnam. Matern Child Nutr 14(1), e12468.

30. Kristjansson E, Francis DK, Liberato S, et al. Food supplementation for improving the physical and psychosocial bealth of socio-economically disadvantaged children aged three months to five years. Cochrane Database Syst Rev 2015(3), CD009924.

31. Pimpin L, Kranz S, Liu E, et al. (2019) Effects of animal protein supplementation of mothers, preterm infants, and term infants on growth outcomes in childhood: a systematic review and meta-analysis of randomized trials. Am J Clin Nutr110(2), 410-429.

32. Sjarif DR, Yuliati K \& Iskandar WJ (2019) Daily consumption of growing-up reduces stunting among Indonesian toddlers. Med J Indonesia 28, 70-76.

33. Lee R, Singh L, van Liefde D, et al. (2018) Milkpowder added to a school meal increases cognitive test scores in Ghanaian children. J Nutr 148(7), 1177-1184.

34. Smithers LG, Golley RK, Mittinty MN, et al. (2013) Do dietary trajectories between infancy and toddlerbood influence IQ in childhood and adolescence? Results from a prospective birth cohort study. PLoS One 8(3), e58904.

35. Miller AL, Lee HJ \& Lumeng JC (2015) Obesity-associated biomarkers and executive function in children. Pediatr Res 77(1-2), 143-7.

36. Bradley R (1988) Using the HOME inventory to assess the family environment. Pediatr Nurs 14(2), 97-102.

37. Double-duty actions for nutrition. Policy Brief. World Health Organization. 2017.

38. World Health Organization. Global nutrition targets 2025. 2014.

39. Smithers LG, Lynch JW, Yang S, et al. (2013) Impact of neonatal growth on IQ and behavior at early school age. Pediatrics 132(1), e53-60.

40. Aubuchon-Endsley NL, Grant SL, Berhanu G, et al. (2011) Hemoglobin, growth, and attention of infants in southern Ethiopia. Child Dev 82(4), 1238-51.

41. Waber DP, Bryce CP, Girard JM, et al. (2014) Impaired IQ and academic skills in adults who experienced moderate to severe infantile malnutrition: a 40-year study. Nutr Neurosci 17(2), 58-64.

42. Sudfeld CR, McCoy DC, Fink G, et al. (2015) Malnutrition and its determinants are associated with suboptimal cognitive, communication, and motor development in Tanzanian children. J Nutr 145(12), 2705-14.

43. Ip P, Ho FKW, Rao N, et al. (2017) Impact of nutritional supplements on cognitive development of children in developing countries: a meta-analysis. Sci Rep 7(1), 10611.

44. Larson LM and Yousafzai AK (2017) A meta-analysis of nutrition interventions on mental development of children under-two in low- and middle-income countries. Matern Child Nutr 13(1). 